\title{
Editorial
}

\section{Unravelling the confusions in prostatic disease}

Why do prostate diseases generate so much controversy? The answer probably lies in the fact that the prostate has long been something of a Cinderella organ. Although prostatic diseases constitute a major source of morbidity and mortality, until recently they have attracted only a fraction of the research funding allocated to, for example, breast or lung cancer. As a consequence, many of the pivotal studies remain to be done.

Things are now beginning to change. In this third issue of Prostate Cancer and Prostatic Diseases some further pieces of the jigsaw are slotted into place. Judd Moul provides us with an elegant review of the increased risk of prostate cancer observed in African American men. Krongrad et al document the disease-specific death risk after external beam ratiotherapy, while Letran and Brawer remind us of the difficulties posed by local recurrence of prostate cancer after this form of treatment.

Although the number of expected life years lost as a result of prostate cancer is modest compared with that of, say, colon cancer, the impact of prostate cancer on quality of life is considerable. The burden of symptoms borne by sufferers, due not only to the disease but also to the treatment required for it, is the subject of two excellent articles in this issue.
Benign prostatic hyperplasia (BPH) is another very important, but under-researched disease area, and one that also impacts substantially on quality of life. But why are some men destined to suffer, while others remain unaffected all their lives? Hammarsten et al in an innovative paper consider possible risk factors for symptomatic BPH. In established $\mathrm{BPH}$ haematuria is a not uncommon occurrence, and one traditionally managed by surgery. In a small, uncontrolled study Holmes et al report that the 5-alpha reductase inhibitor finasteride can be used to treat this, possibly by diminishing the vascularity of the gland. Another paper evaluates the use of the alpha blocker doxazosin in patients with lower urinary tract symptoms due to $\mathrm{BPH}$ in an am. versus pm. dosage regime.

Much remains to be done before all the confusion surrounding prostatic disease is unravelled, but the papers in this issue of the Journal take us some steps in the right direction. Please send us your own contributions for consideration for possible publication. We are all in this together!

Roger Kirby

Howard Scher

Michael Brawer

Correspondence: Dr Roger Kirby, Urology Research Centre, 1st Floor, Ingeleby House, St Georges Hospital, Blackshaw Road, London SW17 ORE. 